\title{
Escala diagramática para a quantificação de Septoria apiicola e Cercospora arracacina em mandioquinha-salsa
}

\author{
Renata M. Mesquini', Kátia R.F. Schwan-Estrada ${ }^{1}$, Cláudia V. Godoy ${ }^{2}$, Rafael A. Vieira ${ }^{1}$, Nestor A.H. \\ Zarate $^{3}$ \& Maria do Carmo Vieira ${ }^{3}$
}

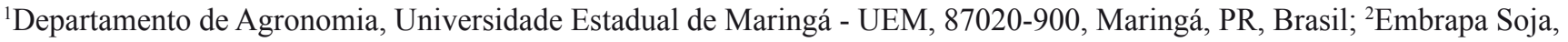
86001-970, Londrina, PR, Brasil; ' ${ }^{3}$ nniversidade Federal da Grande Dourados, Faculdade de Ciências Agrárias, 79804-970, Dourados MS, Brasil

Autor para correspondência: Kátia R.F. Schwan-Estrada, e-mail: schwan@wnet.com.br

\section{RESUMO}

As manchas foliares causadas pelos fungos Septoria apiicola e Cercospora arracacina estão entre as principais doenças foliares da mandioquinha-salsa. O objetivo desse trabalho foi propor uma escala diagramática para quantificar essas doenças, em função da necessidade de obter métodos padronizados para avaliação de doença. Folhas com diferentes níveis de severidade foram coletadas para determinação do limite mínimo e máximo de severidade sendo níveis intermediários determinados de acordo com a "lei do estímuloresposta de Weber-Fechner". A escala foi elaborada contendo sete níveis de severidade $(0,7 ; 2 ; 4 ; 8 ; 17 ; 32$ e $53 \%)$. A validação da escala foi realizada por quatro avaliadores experientes e quatro inexperientes, os quais estimaram com e sem o auxílio da escala, a severidade de 50 folhas com diferentes níveis das doenças. Posteriormente, os valores de severidade real e severidade estimada foram analisados por meio de regressão linear e as estimativas comparadas quanto à acurácia e precisão. Com a utilização da escala, os avaliadores apresentaram melhores níveis de precisão e acurácia e a variância dos erros na quantificação da doença obteve valores dentro dos limites aceitáveis. Dessa forma, a escala proposta mostrou-se adequada para avaliação dessas manchas foliares em mandioquinha-salsa.

Palavras-chave: Arracacia xanthorrhiza, escala diagramática, avaliação de doenças, patometria.

\begin{abstract}
Diagrammatic Scale for Assessment of Septoria apiicola and Cercospora arracacina in Arracacha

Leaf spots, caused by the fungi Septoria apiicola and Cercospora arracacina, are among the most serious leaf diseases that affect arracacha. Since standardized methods for disease evaluation are necessary, this work proposes a diagrammatic scale for assessment of these diseases. Leaves presenting different severity levels were collected and the minimum and maximum severity limits were determined. The intermediary severity levels were determined according to "Weber-Fechner's stimulus response law" and the scale had seven severity levels $(0.7 ; 2 ; 4 ; 8 ; 17 ; 32$ and $53 \%)$. Scale validation was carried out by four experienced raters and four inexperienced raters, who estimated severity with and without the use of the scale, using 50 leaves with different levels of disease. The actual severity and the estimated severity values were analyzed through linear regression, and the estimates were compared for accuracy and precision. By using the scale, the raters obtained higher precision and accuracy levels, and the variance of the absolute errors in the assessment of the disease presented values within acceptable limits. The proposed scale was thus considered appropriate for evaluation of leaf spots in arracacha. Keywords: Arracacia xanthorrhiza, diagrammatic scale, disease assessment, pathometry.
\end{abstract}

O cultivo da mandioquinha-salsa (Arracacia xanthorrhiza Bancr.) no Brasil representa uma área de 7.633 ha, com produtividade média de $9.513 \mathrm{~kg} \mathrm{ha}^{-1}$, e produção anual de 72.616 toneladas. (Santos et al., 2000). No Brasil, 27 gêneros de fungos que causam doenças na cultura da mandioquinha-salsa já foram relatados (Madeira \& Souza, 2004). As espécies Septoria apiicola Speg. (sin. Septoria apii Chester) e Cercospora arracacina Chupp causadoras de manchas foliares são de ampla ocorrência nos campos de produção (Henz, 2002). Os seus sintomas consistem de pequenas manchas castanhas com lesões centrais, onde se localizam as estruturas de esporulação, sendo picnídios para S. apiicola e conidióforos livres para C. arracacina.
Em estudos de epidemiologia quantitativa, as escalas diagramáticas têm sido fundamentais para melhorar a acurácia e a precisão nas avaliações e, assim, proporcionar resultados mais concisos (Spósito et al., 2004). No caso de doenças foliares, a severidade é a variável mais utilizada, sendo que sua avaliação é normalmente feita de forma subjetiva por meio de análises visuais. Por conseguinte, as escalas diagramáticas tornam-se ferramentas fundamentais em tais estudos para auxiliar o avaliador e reduzir a subjetividade (Godoy et al., 1997, Leite \& Amorim, 2002).

$\mathrm{Na}$ construção de uma escala, alguns aspectos importantes devem ser considerados, como: a) os limites superiores e inferiores da escala devem corresponder, 
respectivamente, à máxima e mínima intensidade da doença observada no campo; b) os sintomas devem ser os mais próximos possíveis dos observados em plantas e; c) nos níveis intermediários de doença devem ser consideradas as limitações de acuidade do olho humano definidas pela "lei de estímulos-resposta de Weber-Fechner", na qual a acuidade visual é proporcional ao logarítmo da intensidade do estímulo (Nutter \& Schultz, 1995).

As escalas diagramáticas têm sido desenvolvidas para utilização na estimativa da severidade de doenças fúngicas em várias culturas, tais como feijoeiro (Godoy et al., 1997), inhame (Michereff et al., 2000), melancia (Halfeld-Vieira et al., 2006), videira (Angelotti et al., 2008), mamoneira (Sussel et al., 2009), entre outras. Entretanto, para a quantificação de manchas foliares causadas por $S$. apiicola e C. arracacina em mandioquinha-salsa, não há escalas que contemplem esta necessidade. Desta forma, este trabalho teve como objetivo a elaboração e a validação de uma escala diagramática para quantificação da severidade neste patossistema.

Para a elaboração da escala diagramática, foram coletadas 90 folhas em campo de produção da cultivar 'Amarela de Carandaí', com diferentes níveis de severidade e de forma aleatória. Estas folhas foram digitalizadas em scanner com resolução de 300 dpi e, em seguida, determinouse a porcentagem de área lesionada, por meio do programa Quant (Vale et al., 2003). Os valores mínimo e máximo de severidade foram considerados, respectivamente, o limite inferior e o limite superior de doença e, representados nos níveis iniciais e finais da escala. Os níveis intermediários da escala foram determinados de acordo com a acuidade da visão humana definida pela "lei do estímulo-resposta de Weber - Fechner" (Horsfall \& Cowling, 1978).
A validação da escala foi realizada a partir de duas avaliações de severidade em 50 folhas com diferentes níveis de severidade, digitalizadas por meio do scanner. Para isso, oito avaliadores, sendo quatro desses com experiência em quantificação de doenças, e quatro sem experiência, estimaram a severidade da doença na primeira avaliação sem o auxílio da escala, e na segunda avaliação, utilizando a escala proposta neste trabalho.

A precisão e a acurácia das estimativas visuais de cada avaliador foram determinadas por meio da regressão linear entre a severidade real (variável independente) e a severidade estimada (variável dependente) onde determinouse o intercepto $(a)$, e o coeficiente angular da reta $(b)$. A acurácia foi verificada por meio do teste $t$ averiguando as hipóteses Ho: $a=0$ e Ho: $b=1$, ao nível de significância $\mathrm{p}$ $=0,01$. A precisão foi determinada pelo coeficiente de determinação da regressão $\left(\mathrm{R}^{2}\right)$ e pela variância dos erros absolutos entre a severidade real e a severidade estimada nas avaliações com e sem a escala, e para inferir com relação ao auxílio proporcionado pela escala (Godoy et al., 1997, Nutter \& Schultz, 1995). As análises de regressão foram realizadas por meio do programa SAS 9.1 (SAS Institute, 2002).

A escala diagramática proposta neste trabalho teve como nível máximo 53\% de severidade e mínimo 0,7\%. Os valores intermediários calculados foram 2, 4, 8, 17 e $32 \%$ (Figura 1). Valores acima de 53\% não foram incluídos, uma vez que levam as folhas à senescência. Para a maioria dos avaliadores, os valores de severidade estimados com o auxílio da escala ficaram mais próximos dos valores da severidade real, quando comparados com os valores obtidos sem a utilização da escala (Figuras 2 e 3). Esta proximidade entre os valores estimados e os reais, determina a acurácia
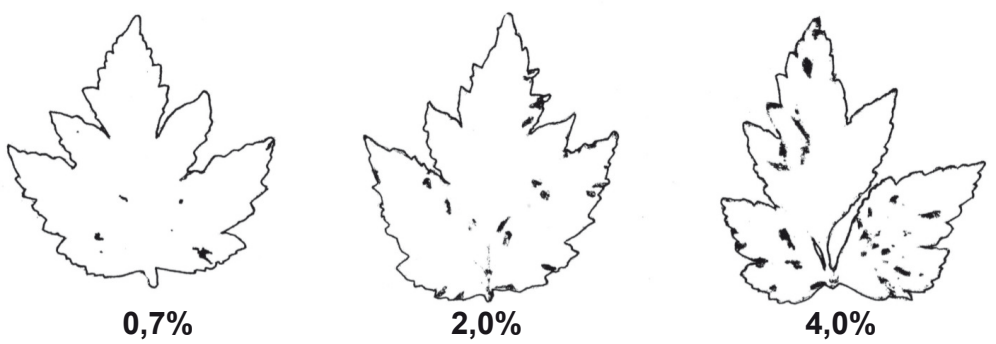

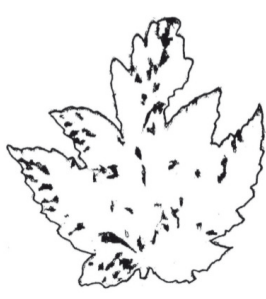

$8,0 \%$

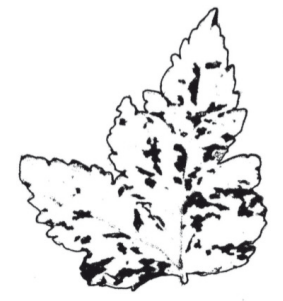

$17 \%$

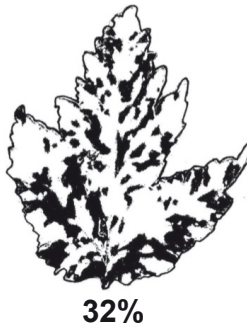

$32 \%$

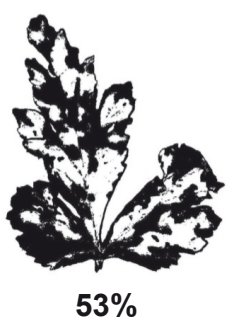

FIGURA 1 - Escala diagramática para quantificação da severidade de manchas foliares, causadas por Septoria apiicola e Cercospora arracacina em mandioquinha-salsa (Arracacia xanthorrhiza). Os valores representam a porcentagem da área foliar necrosada. 

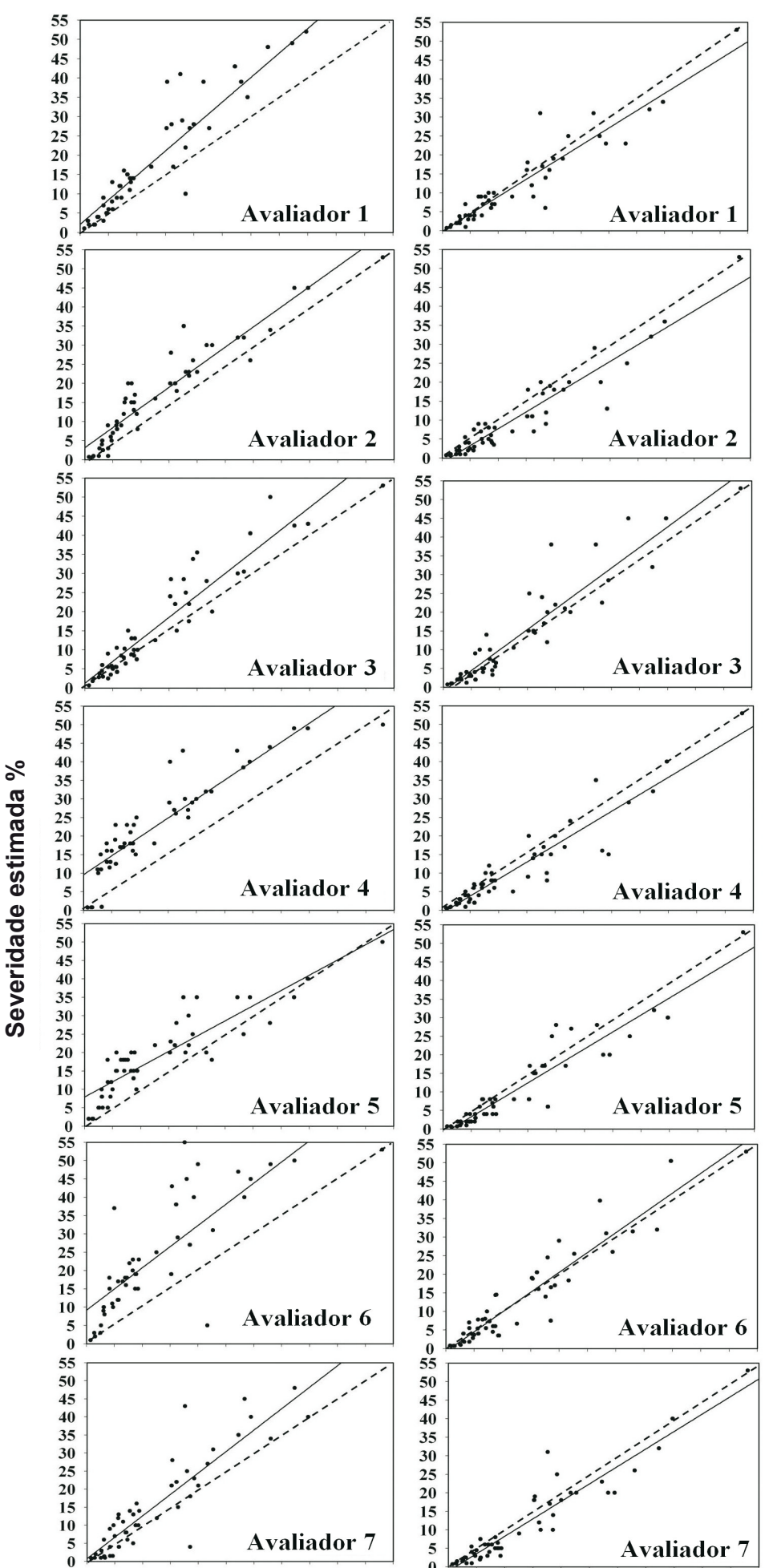

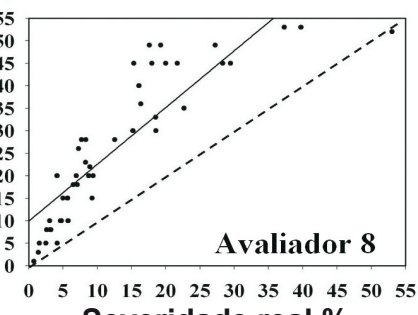

Severidade real \%
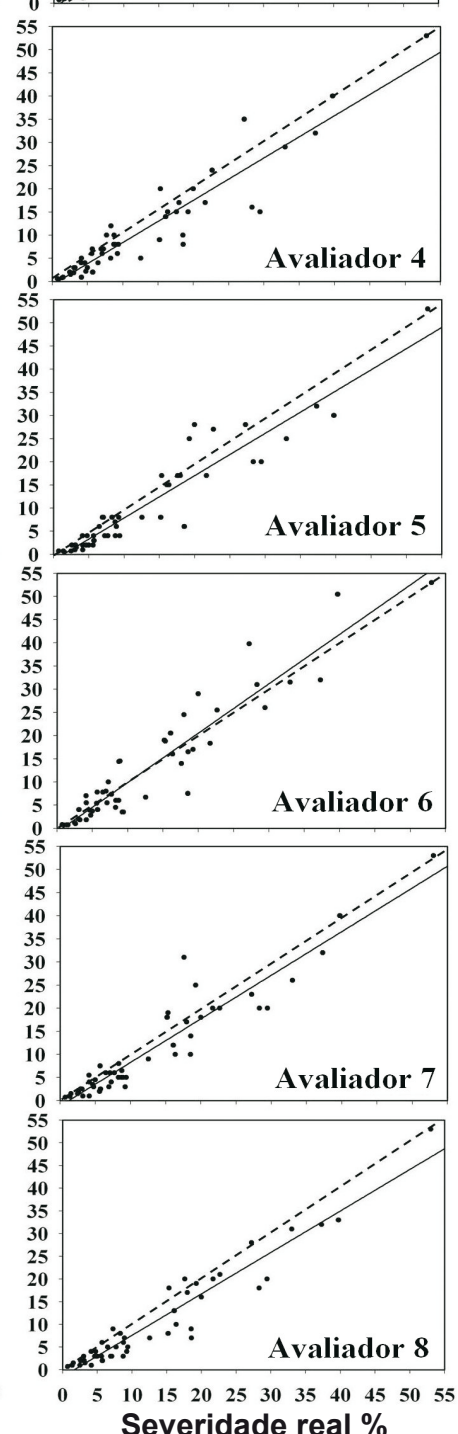

FIGURA 2 - Severidade estimada sem o auxílio da escala diagramática (à esquerda) e severidade estimada com o auxílio da escala diagramática proposta (à direita), para a manchas foliares (Septoria apiicola e Cercospora arracacina (pontos cheios) e linhas de regressão obtidas entre a severidade real e a estimada (linha preta). A linha pontilhada representa a severidade real. Avaliadores 1 a 4 são experientes; avaliadores 5 a 8 são inexperientes. 


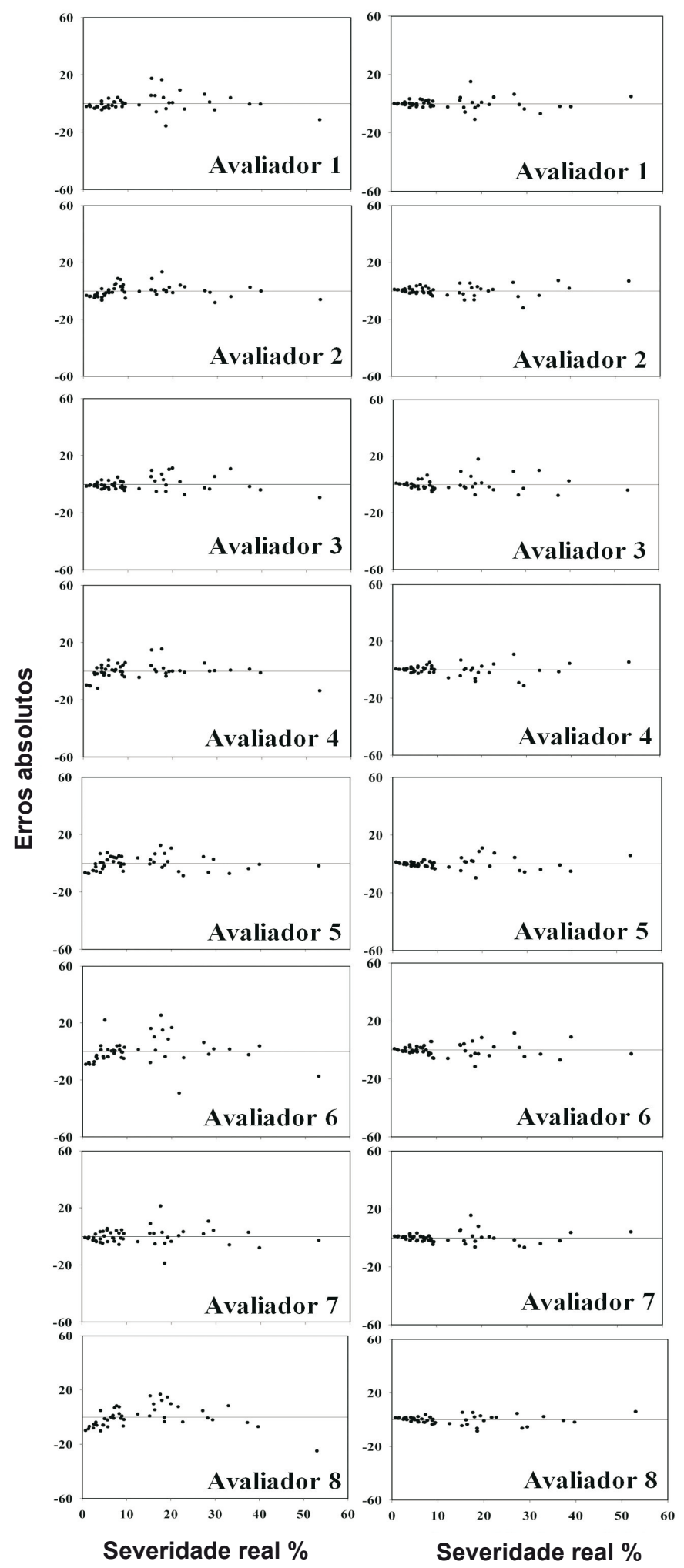

FIGURA 3 - Erros absolutos das análises de regressão linear entre valores de severidade real e estimada, sem o auxílio da escala diagramática (à esquerda), e com o auxílio da escala proposta (à direita) para manchas foliares em mandioquinhasalsa (Arracacia xanthorrhiza). Avaliadores 1 a 4 são experientes; avaliadores 5 a 8 são inexperientes. 
das avaliações, termo este definido como a exatidão de uma medida isenta de erros sistemáticos. Desta forma, avaliadores considerados acurados têm a média das estimativas de severidade próximas da média real. Para isto ocorrer, de acordo com Nutter Jr. et al. (1993), a inclinação da regressão linear $(b)$ deve ser igual a um 1 , sem desvios sistemáticos e o intercepto $(a)$ deve ser igual a zero (0). Constatou-se que para todos os avaliadores os valores de $b$ foram significativamente iguais a 1 , não incorrendo em erros sistemáticos (Tabela 1). $\mathrm{O}$ avaliador 8 , pode ser considerado o menos acurado, pois os valores de $a$ foram significativamente diferentes de 0 . Todos os avaliadores, foram mais acurados com a utilização da escala, fato este comprovado pelos valores do intercepto e do coeficiente angular estarem mais próximos de 0 e 1 respectivamente.

Não foi observado tendências à superestimativa e a subestimativa com a utilização da escala. Sem a utilização da escala, tanto os avaliadores experientes quanto os inexperientes tiveram uma tendência a superestimativa, porém com a utilização da mesma, houve uma melhora na precisão, evitando erros sistemáticos (Figura 3). Outros estudos de validação de escalas diagramáticas indicaram que a tendência em superestimar valores é mais freqüente (Parker et al., 1995; Leite \& Amorim, 2002; Spósito et al., 2004). Além da acurácia, a precisão, definida como a exatidão de uma operação onde há rigor ou refinamento na medida (Bergamin Filho \& Amorim, 1996) é um fator a ser considerado na validação de escalas diagramáticas.

Navalidaçãodaescala,tantoosavaliadoresexperientes quanto os inexperientes apresentaram maior precisão nas avaliações quando utilizaram a escala diagramática. Isso foi verificado pelo aumento do coeficiente de determinação $\left(\mathrm{R}^{2}\right)$ quando se compara os valores na avaliação sem a escala e com o auxilio da mesma, sendo que para os avaliadores experientes foi observado menor acréscimo em termos de precisão em relação aos inexperientes, nos quais a escala possibilitou maior incremento da precisão (Tabela 1). Os coeficientes de determinação com o uso da escala variaram entre 0,88 e 0,92 , sendo semelhantes ou superiores aos níveis verificados em outros estudos utilizando escalas diagramáticas (Rodrigues et al., 2002; Martins et al., 2004; Andrade et al., 2005) e estão próximos a 0,95, considerado limiar desejável por Kranz (1988).

Além do $\mathrm{R}^{2}$, a boa precisão dos avaliadores foi confirmada quando se efetuou a análise dos erros absolutos, que consideram a diferença entre a severidade estimada e a real. Observa-se que nas avaliações realizadas pelos avaliadores inexperientes, sem o auxilio da escala, os erros absolutos ultrapassaram 20\% (Figura 3). Já com o auxilio desta, os erros absolutos se concentraram entre 5 e $10 \%$, sendo considerados níveis aceitáveis, conforme critérios adotados por outros autores (Leite \& Amorim, 2002; Spósito et al., 2004). É importante ressaltar que a presença de certo nível de erro absoluto nas mensurações pode ser compensada pela rapidez e padronização quando se utiliza escala diagramática (Stonehouse, 1994).

As análises estatísticas indicam a eficiência da escala diagramática proposta neste trabalho sugerindo sua utilização como uma ferramenta bastante oportuna, de fácil utilização e, que pode auxiliar avaliadores experientes e inexperientes na quantificação de manchas foliares causadas por S. apiicola e C. arracacina em mandioquinha-salsa.

TABELA 1 - Acurácia e precisão representadas pelo intercepto $(a)$, coeficientes angulares $(b)$ e coeficientes de determinação $\left(\mathrm{R}^{2}\right)$ obtidos nas regressões entre severidade real (variável independente) e severidade estimada (variável dependente) de manchas foliares em mandioquinha-salsa (Arracacia xanthorrhiza), por oito avaliadores (1 ao 4 experientes e 5 ao 8 inexperientes), com e sem o auxílio da escala diagramática

\begin{tabular}{|c|c|c|c|c|c|c|}
\hline \multirow[t]{2}{*}{ Avaliador } & \multicolumn{3}{|c|}{ Sem escala } & \multicolumn{3}{|c|}{ Com escala } \\
\hline & $\mathbf{R}^{2}$ & $a$ & $b$ & $\mathbf{R}^{2}$ & $a$ & $b$ \\
\hline \multicolumn{7}{|c|}{ Experientes } \\
\hline 1 & 0,87 & 2,11 & 1,26 & 0,89 & $-0,10$ & 0,91 \\
\hline 2 & 0,89 & $3,17 * *$ & 1,05 & 0,90 & $-1,15$ & 0,89 \\
\hline 3 & 0,90 & 1,26 & 1,14 & 0,88 & $-1,02$ & 1,09 \\
\hline 4 & 0,81 & $9,59 * *$ & 1,02 & 0,88 & 0,71 & 0,91 \\
\hline \multicolumn{7}{|c|}{ Inexperientes } \\
\hline 5 & 0,78 & $7,94 * *$ & 0,82 & 0,88 & $-1,24$ & 0,91 \\
\hline 6 & 0,67 & $9,18 * *$ & 1,15 & 0,89 & $-0,78$ & 1,06 \\
\hline 7 & 0,85 & 0,76 & 1,19 & 0,90 & $-1,15$ & 0,94 \\
\hline 8 & 0,77 & $9,94 * *$ & 1,26 & 0,92 & $-1,51 * *$ & 0,91 \\
\hline
\end{tabular}

** situações nas quais as hipóteses $a=0$ ou $b=1$ foram rejeitadas pelo teste $t$ a $1 \%$ de significância. 


\section{REFERÊNCIAS BIBLIOGRÁFICAS}

Andrade GCG, Alfenas AC, Máfia RG, Máffia LA, Gonçalves RC (2005) Escala diagramática para avaliação da severidade da mancha foliar do eucalipto causada por Quambalaria eucalypti. Fitopatologia Brasileira 30:504- 509.

Angelotti F, Scapin CR, Tessmann DJ, Vida JB, Oliveira RR, Canteri MG (2008) Diagrammatic scale for assessment of grapevine rust. Tropical Plant Pathology 33:439-443.

Godoy CV, Carneiro SMTPG, Iamauti MT, Dalla Pria M, Amorim L, Berger RD, Bergamin Filho A (1997) Diagrammatic scales for bean disease: development and validation. Zeitschrift für Pflanzenkrankheiten und Pflanzenschutz 104:336-345.

Halfeld-Vieira BA, Nechet KL (2006) Elaboração e validação de escala diagramática para avaliação da mancha-de-cercospora em melancia. Fitopatologia Brasileira 31:46-50.

Henz GP (2002) Doenças da mandioquinha-salsa e sua situação atual no Brasil. Horticultura Brasileira 20:135-144.

Horsfall JG, Cowling EB (1978) Pathometry: The measurement of plant disease. In: Horsfall JG, Cowling EB (Eds.) Plant Disease: An Advanced Treatise - How Disease Develops in Populations. New York NY. Academic Press. pp. 119-136.

Kranz J (1988) Measuring Plant Disease. In: Kranz, J, Rotem J (Eds.) Experimental Techniques in Plant Disease Epidemiology. Berlin. Springer Verlag. pp. 35-50.

Leite MVBCL, Amorim L (2002) Elaboração e validação de escala diagramática para mancha de Alternaria em girassol. Summa Phytopathologica 28:14-19.

Madeira NR, Souza RJ (2004) Mandioquinha - salsa: alternativa para o pequeno produtor. Lavras MG. Editora Universidade Federal de Lavras.

Martins MC, Guerzoni RA, Câmara GMS, Mattiazi P, Lourenço AS, Amorim L (2004) Escala diagramática para a quantificação do complexo de doenças foliares de final de ciclo em soja. Fitopatologia Brasileira 29:179-184.
Michereff SJ, Maffia LA, Noronha MA(2000) Escala diagramática para avaliação da severidade da queima das folhas de inhame. Fitopatologia Brasileira 25:612-619.

Nutter Jr FW, Schultz PM (1995) Improving the accuracy and precision of disease assessments: selection of methods and use of computer-aided training programs. Canadian Journal of Plant Pathology 17:174-184.

Parker SR, Whelan MJ, Royle DJ (1995). Reliable measurement of disease severity. Aspects of Applied Biology 43:205-214

Rodrigues JCV, Nogueira NL, Machado MA (2002) Elaboração e validação de uma escala diagramática para leprose dos citros. Summa Phytopathologica 28:192-196.

Rohrbach KG, Schmitt HD. Pineapple. In: Ploetz RC, Zentmyer GA, Nishijima WT, Rohrbach KG, Ohr HD. (1994) (Eds). Compendium of tropical fruit diseases. Saint Paul MN. APS Press. pp. $45-55$.

Santos FF, Costa GP, Macedo P, Krieck RS (2000) Mandioquinhasalsa no agronegócio do estado do Paraná. Curitiba PR. Informação Técnica no. 51.

SAS Institute (2002). User's guide: statistics, version 9.1. Cary. SAS Institute.

Spósito MB, Amorim L, Belasque Junior J, Bassanezi RB, Aquino R (2004) Elaboração e validação de escala diagramática para avaliação da severidade da mancha preta em frutos cítricos. Fitopatologia Brasileira 29:81-85.

Stonehouse J (1994). Assessment of Andean bean disease using visual keys. Plant Pathology 43:519-527.

Sussel AAB, Pozza EA, Castro HA (2009) Elaboração e validação de escala diagramática para avaliação da severidade do mofo cinzento em mamoneira. Tropical Plant Pathology 34:186-191.

Vale FXR, Fernandes Filho EI, Liberato JR (2003) QUANT. A software for plant disease severity assessment. In: $8^{\text {th }}$ International Congress of Plant Pathology, Christchurch New Zealand, p. 105.

TPP 9004 - Recebido 12 Janeiro 2009 - Aceito 12 Agosto 2009 Editor de Seção: Lilian Amorim 